\title{
Statistical Analysis of Pulsed Micro-discharges and Ozone Generation in Dielectric Barrier Discharges
}

\author{
Guangming Huang, Yingjia Zhou, Tao Wang, Igor V. Timoshkin, Mark P. Wilson, Scott J. MacGregor, \\ Martin J. Given
}

\begin{abstract}
Pulsed micro-discharges produced by dielectric barrier discharges (DBDs) in a sub-millimeter gap were investigated under 200 cycles of sinusoidal ac voltage at $5 \mathrm{kHz}$ in this work. The impulsive current in the external circuit was accurately measured by an oscilloscope with a bandwidth of $2.5 \mathrm{GHz}$ and maximum sampling rate of $40 \mathrm{GS} / \mathrm{s}$ to calculate the filamentary current in the discharge gap. The amplitude, pulse duration and transferred charge of a single filamentary current and the micro-discharge energy acquired over the 200 voltage cycles were statistically analyzed for different discharge gaps and gas pressures. The micro-discharge parameters and ozone generation efficiencies for different conditions were compared. The ozone production efficiency was found to increase with increasing pressure from 1 bar absolute to 2 bar absolute, and the gap length from $0.2 \mathrm{~mm}$ to $0.5 \mathrm{~mm}$. The maximum ozone production efficiency achieved in the work was $217 \mathrm{~g} / \mathrm{kWh}$, with a gap length of $0.5 \mathrm{~mm}, 2.0 \mathrm{bar}$ absolute pressure, and an applied voltage of 5.5 $\mathrm{kV}$ at $5 \mathrm{kHz}$.
\end{abstract}

Index Terms-Dielectric barrier discharges, filamentary current, micro-discharges, reduced electric field and ozone generation

\section{INTRODUCTION}

$\mathrm{O}^{2}$ ZONE is a strong oxidant and widely used in water treatment, pulp bleaching and the $\mathrm{DeNO}_{\mathrm{x}}$ process. Dielectric barrier discharges have been employed in industry as the most efficient method for ozone generation, though more than $80 \%$ of the electrical energy is dissipated as heat in the discharge [1]. Optimization of pulsed micro-discharges is an important area of research in attempts to increase ozone generation efficiency [2]. However, numerous pulsed micro-discharges in DBDs take place randomly in the discharge gap, making their electrical characterization difficult in two aspects: measuring the filamentary current, and understanding its distribution over the applied voltage cycles. Over the last 30 years, much research has been dedicated to the electrical characterization of micro-discharges. Two approaches to measure the filamentary current have been identified in the literature: direct measurement by incorporation

This paper was submitted for review on 28/12/2015.

All the authors are with the department of electronic and electrical engineering, University of Strathclyde, Royal College Building, 204 George Street, Glasgow, UK, G1 1XW (email: g.huang@strath.ac.uk). of a well-shielded fast current probe into the ground electrode to measure the current of an individual micro-discharge in the gap [3-5]; and indirectly by measuring the external impulsive current and then calculating the filamentary current, $I_{f}$, in the gap [6-9], as shown in (1).

$$
I_{f}=\left(1+\frac{C_{g}}{C_{d}}\right) I_{t}-C_{g} \frac{d V_{a}}{d t}
$$

where $C_{g}$ and $C_{d}$ are the capacitances of the gap and the dielectric barrier respectively; $I_{t}$ is the total external current and $V_{a}$ is the applied voltage.

Disadvantages associated with direct measurement include the fact that it can only provide information on the filamentary current in one specific location, and it is difficult to construct a ground electrode to incorporate a well-shielded current probe without undesirable modification of the original field distribution. J. Dřímal et al. [6] and S. Liu et al. [7] derived an equation relating the filamentary current to the applied voltage and external impulsive current. In the work done by J. Dř́mal et al. [6] a term incorporating the derivative of applied voltage was neglected, however, leading to inaccurate calculation of the filamentary current and the transferred charge, since there are always abrupt jumps in the applied voltage when a discharge occurs [10]. In the work by S. Liu et al. [7], both the external current and the derivative of applied voltage were considered, but only the calculated filamentary current waveform in one cycle was presented; the statistical distribution of the filamentary current over several voltage cycles was not discussed. N. Jidenko et al. provided a statistical distribution of the electrical characteristics over 500 micro-discharges in [9], but only the factor $\left(1+C_{g} / C_{d}\right)$ was taken into account for calculation of the filamentary current in the gap, the derivative of applied voltage being neglected.

In the present work, the instantaneous applied voltage and the impulsive current in the external circuit were measured by an oscilloscope with a bandwidth of $2.5 \mathrm{GHz}$ and maximum sampling rate of $40 \mathrm{GS} / \mathrm{s}$. The oscilloscope memory allows the acquisition of 200 cycles of voltage and current information with sampling rate of $5 \mathrm{G} / \mathrm{s}$ when operating at $5 \mathrm{kHz}$. Subsequent processing of the recorded data using Matlab revealed the statistical distribution of important electrical 
parameters of pulsed micro-discharges during the 200 voltage cycles. In contrast to previous studies in the literature, the statistical distribution of filamentary current, considering both the factor $\left(1+C_{g} / C_{d}\right)$ and the derivative of applied voltage, has been analyzed in this paper. To optimize the pulsed micro-discharges in the barrier discharge for improved ozone generation efficiency, ozone generation data was recorded simultaneously during the filamentary current measurement. The relationship between the reduced electric field, filamentary current and ozone generation efficiency for different discharge gaps and gas pressures was investigated, and the results are presented and discussed herein.

\section{EXPERIMENTAL SET-UP AND METHODOLOGY}

\section{A. Experimental Set-up}

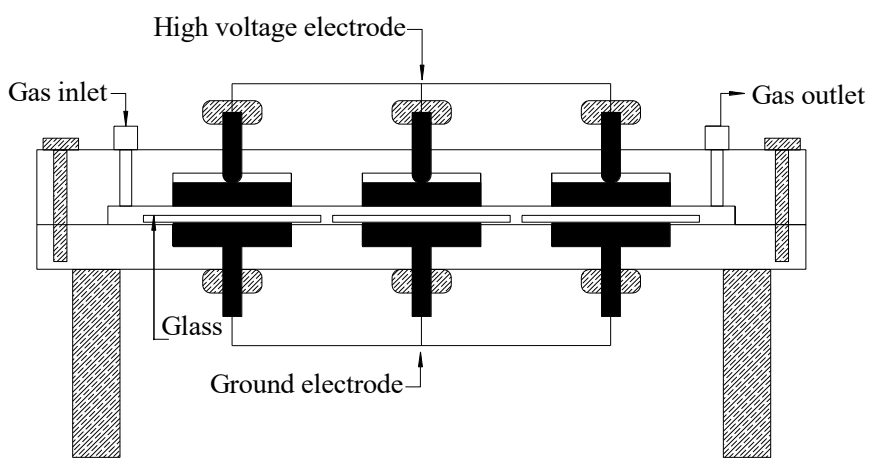

(a)

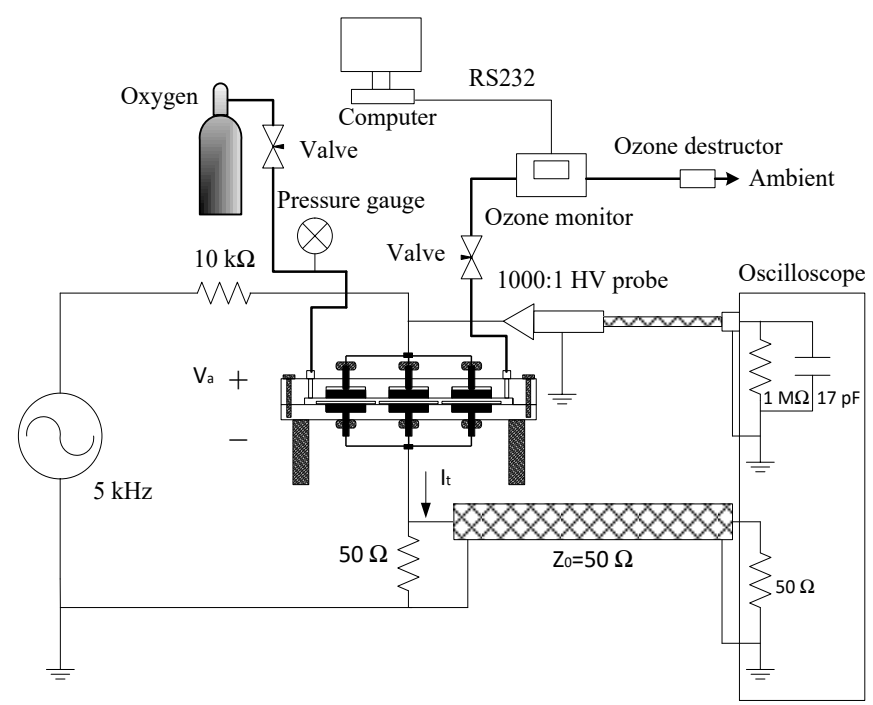

(b)

Fig. 1. Schematic diagram of ozone reactor (a) and experimental set-up (b)

Fig. 1 shows a schematic diagram of the ozone reactor and experimental set-up used in the study. The DBD ozone generator consists of three pairs of electrodes, with a dielectric barrier composed of soda-lime glass of thickness $0.5 \mathrm{~mm}$. Each electrode plate has dimensions of $50 \mathrm{~mm}$ length, and $30 \mathrm{~mm}$ width. The discharge gap was constructed in a PTFE chamber and its length can be accurately controlled from $0.2 \mathrm{~mm}$ to 0.6 $\mathrm{mm}$. The ozone generator was energized by 200 cycles of a sinusoidal voltage with amplitude of $5.5 \mathrm{kV}$ at a frequency of 5 $\mathrm{kHz}$. The ac power supply employed was a Pacific 112AMX; the output voltage of $300 \mathrm{~V}$ being stepped up to $5.5 \mathrm{kV}$ by a high-frequency transformer. Experiments were conducted in an oxygen environment, with absolute pressure from 1 bar to 2 bar, and the ambient temperature of $25{ }^{\circ} \mathrm{C}$. The internal gas pressure was controlled through two needle valves located before and after the ozone reactor. The flow rate was fixed at $0.5 \mathrm{~L} / \mathrm{min}$ in all experiments. The external current was measured through a $50 \Omega$ coaxial cable (RG405), with a bandwidth of $18 \mathrm{GHz}$. The voltage across the ozone reactor was measured using a LeCroy PPE6kV high-voltage probe (400 $\mathrm{MHz}$ ). The measurement data were acquired by an oscilloscope (LeCroy WaveRunner $625 \mathrm{Zi}$ ) with a bandwidth of $2.5 \mathrm{GHz}$ and maximum sampling rate of $40 \mathrm{GS} / \mathrm{s}$.

The ozone concentration was measured using an ozone monitor (BMT964) and the data was transferred to a computer for real-time integration, to calculate the total ozone production during the application of 200 voltage cycles. The discharge power was determined by integration of the instantaneous product of the digitized voltage and current signals from the oscilloscope. The discharge voltage of the gap was determined through the Lissajous figure [11], measured separately using a 220-nF measuring capacitor.

\section{B. Methodology}

A transient sinusoidal voltage of 200 cycles was employed in the work. The choice of applying 200 voltage cycles fulfills the requirements as below:

- It reduces the heating effects in the ozone-synthesis process.

- Electrical measurement data can be acquired and recorded at a high sampling rate $(\geq 5 \mathrm{GS} / \mathrm{s})$.

The parameters measured and recorded during the application of 200 voltage cycles included the applied voltage, $V_{a}$, and the total current, $I_{t}$, in the external circuit, and the ozone concentration as a function of time. The measured data were processed using Matlab to obtain the electrical parameters of each filament and their statistical distributions. The numerical treatments in this work are descripted as below:

\section{1) Separation of the impulsive current}

The measured current in the external circuit consists of two components: displacement current of $5 \mathrm{kHz}$; and impulsive current with duration of the order of ns. A high-pass filter with lower cut-off frequency of $10 \mathrm{kHz}$ was used in Matlab to separate the impulsive current component.

\section{2) Calculation of the filamentary current in the gap}

The filamentary current in the discharge gap was calculated using (1). The high sampling rate of the oscilloscope enabled 
the derivative of the applied voltage $V_{a}$ to be obtained numerically using Matlab.

\section{3) Calculation of the micro-discharge energy}

The energy dissipated by the micro-discharge $\left(\mathrm{W}_{0}\right)$ in the gap is equal to the electrical energy injected into the DBD reactor, assuming that there is no dielectric loss, as shown in (2):

$$
\int_{0}^{t} I_{t}(\tau) V_{a}(\tau) \mathrm{d} \tau=\int_{0}^{t} I_{f}(\tau) V_{g}(\tau) \mathrm{d} \tau
$$

where $V_{g}$ is the gap voltage and $t$ is the duration of the micro-discharge.

4) Statistical treatment of the filamentary current and micro-discharge energy over a representative 25 cycles

Limited by the maximum acquisition memory of the oscilloscope, 25 cycles of voltage and current waveforms at the sampling rate of $5 \mathrm{GS} / \mathrm{s}$ were acquired. The number of discharges per cycle $(n)$, the amplitude of the filamentary current $\left(I_{p}\right)$, the duration of the filamentary current pulse $\left(t_{d}\right)$, the transferred charge associated with a filament $(Q)$, and the individual micro-discharge energy $\left(W_{0}\right)$ over the representative 25 cycles were statistically analyzed.

\section{5) Reduced electric field calculation}

The reduced electric field, $E / N$, is an important quantity that determines the electron energy distribution function and reaction rate coefficients [12-14]. It is the interest of this work to investigate the characteristics of filamentary current and ozone generation efficiency over a range of $E / N$ values, calculated using (3):

$$
E / N=V_{g} /\left(d_{g} \cdot N\right)
$$

where $N$ is the gas density calculated from the ideal gas equation [15], and $d_{g}$ is the gap length. The reduced electric field $E / N$ as a function of gap length and gas pressure were investigated in the study.

\section{6) Ozone generation efficiency calculation}

The ozone concentration was monitored in real-time via a computer-based system. Ozone generation efficiency was calculated as the integral of the ozone produced over 200 cycles divided by the discharge energy over 200 cycles.

\section{RESULTS}

\section{A. Filamentary current pulse statistics}

Fig. 2 shows the waveforms of the applied voltage and external impulsive current measured at discharge. The filamentary current was calculated using (1), and plotted in Fig. 3. As can be seen in Fig. 3, $t_{d}$ is the filamentary current duration, defined as the time interval starting from where the current rises from zero to where the current falls to zero. $I_{p}$ is the amplitude of the filamentary current pulse, and $Q$ is the transferred charge which is equal to the area integrated over the time interval defined by $t_{d}$.

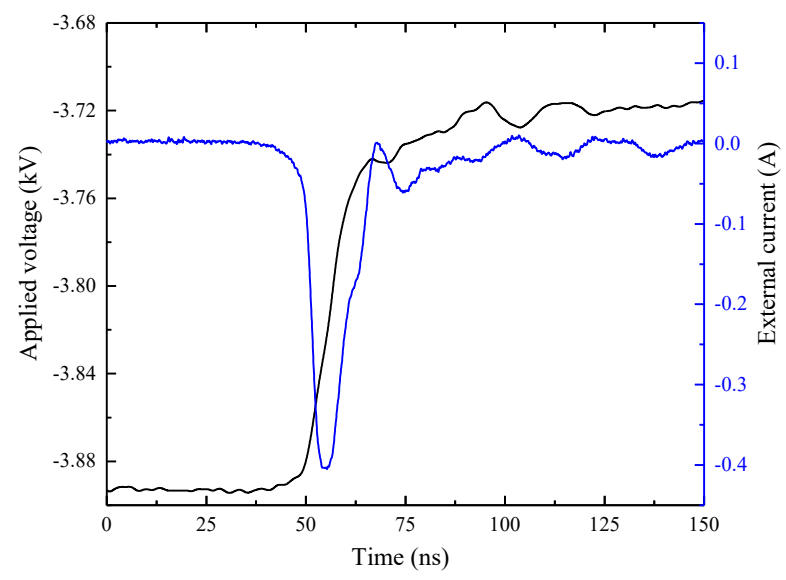

Fig. 2. Applied voltage and external impulsive current

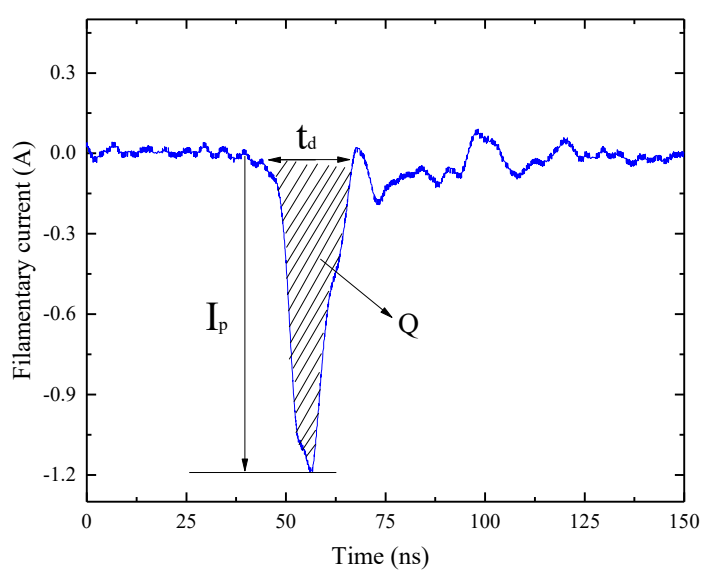

Fig. 3. Calculated filamentary current in the gap

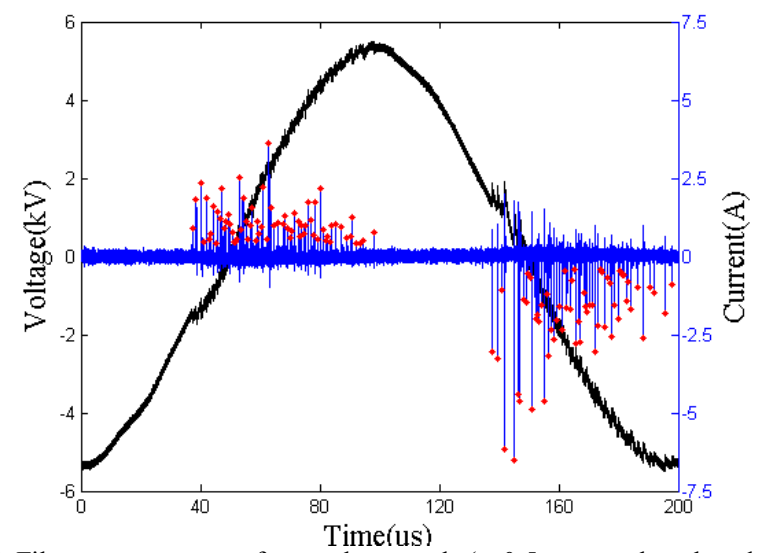

Fig. 4. Filamentary current of one voltage cycle (at $0.5 \mathrm{~mm}$ gap length and $1 \mathrm{bar}$ absolute, with applied voltage $5.5 \mathrm{kV}$ at $5 \mathrm{kHz}$ )

Fig. 4 shows the filamentary current of one voltage cycle, obtained when the peak voltage applied was $5.5 \mathrm{kV}$ and the gap distance was $0.5 \mathrm{~mm}$ at the absolute pressure of 1 bar. The detected filamentary currents are marked using red dots on the Fig. 4. The detection limit applied was $\pm 0.2 \mathrm{~A}$. The important parameters of individual filamentary currents, $I_{p}, t_{d}, Q$ and $W_{0}$, over the representative 25 cycles were statistically analyzed and the histograms of these parameters are presented in Fig. 5 


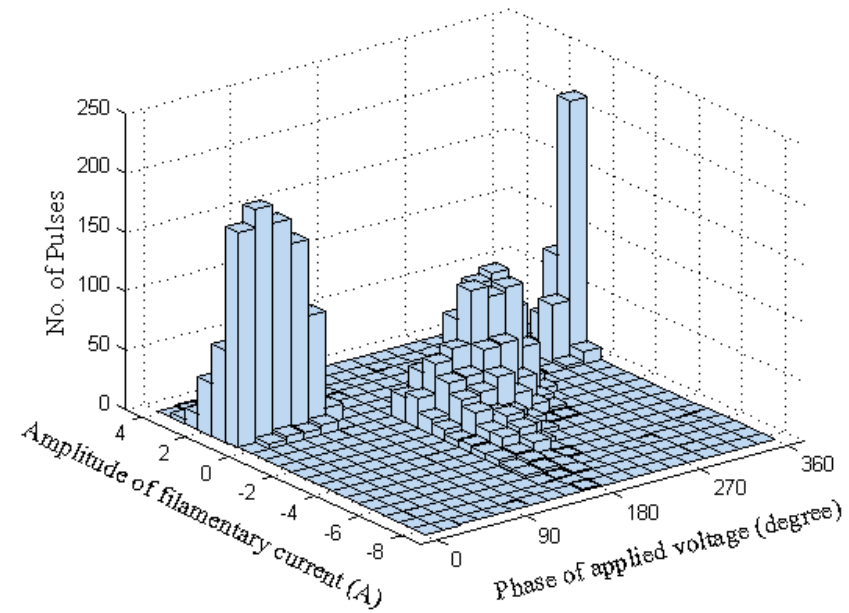

Fig. 5. Histogram of amplitude of filamentary current $I_{p}$ (at $0.5 \mathrm{~mm}$ gap length and 1 bar absolute)

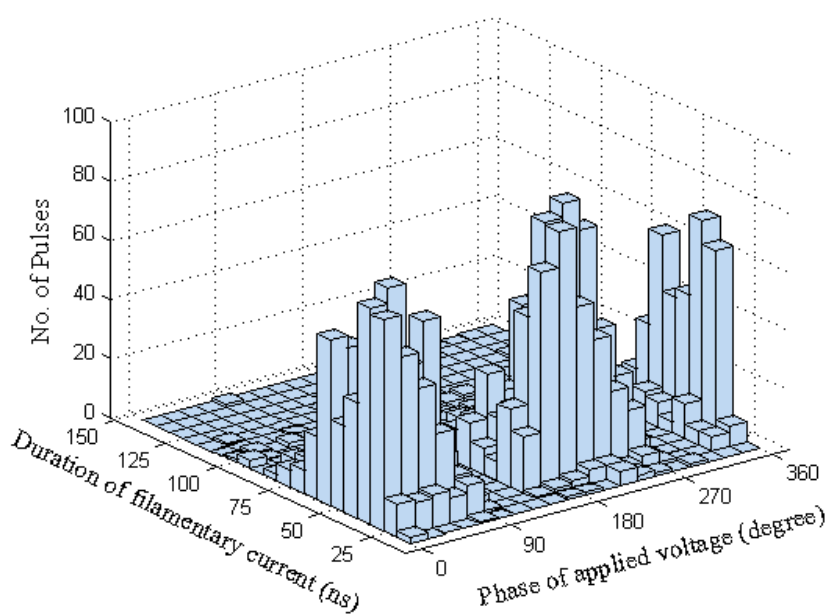

Fig. 6. Histogram of duration of filamentary current $t_{d}$ (at $0.5 \mathrm{~mm}$ gap length and 1 bar absolute)

to Fig. 8. In Fig. 5, the amplitude of positive filamentary currents demonstrates more consistency than that of negative filaments. The amplitudes of most positive current pulses are about $1 \mathrm{~A}$, and few are larger than $2 \mathrm{~A}$. The amplitudes of negative current pulses are widely spread and up to $6 \mathrm{~A}$. It can be observed from Fig. 5 that the current pulses measured at the start of the negative voltage half-cycle (phase angle of $180^{\circ}$ ) have higher amplitudes, whereas the current pulses occurring at around $270^{\circ}$ have relatively consistent amplitude of about $1 \mathrm{~A}$. The corresponding charge transfer is presented in Fig. 7. The statistical distribution of charge is similar to that of current pulse amplitude, which shows that the charge transferred in negative filamentary currents is spread more widely than that of the positive filaments, and the higher charge transfer took place at around $180^{\circ}$. Different from the wide range of current amplitudes, the current duration shows a narrow distribution for both positive and negative half-cycles, as shown in Fig. 6. Most of the current pulse durations are around $46 \mathrm{~ns}$. Fig. 8 gives the distribution of energy dissipated by micro-discharges. It is found that the micro-discharges occurring at the beginning of the discharge period, where the voltage phase angle is $0^{\circ}$ and $180^{\circ}$, consumes similar amount of energy; but when the voltage

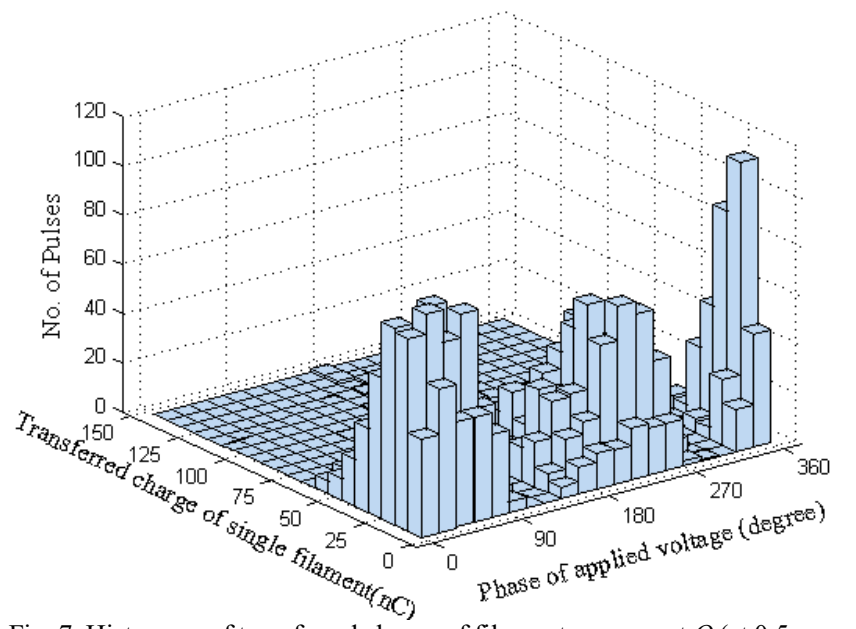

Fig. 7. Histogram of transferred charge of filamentary current $Q$ (at $0.5 \mathrm{~mm}$ gap length and 1 bar absolute)

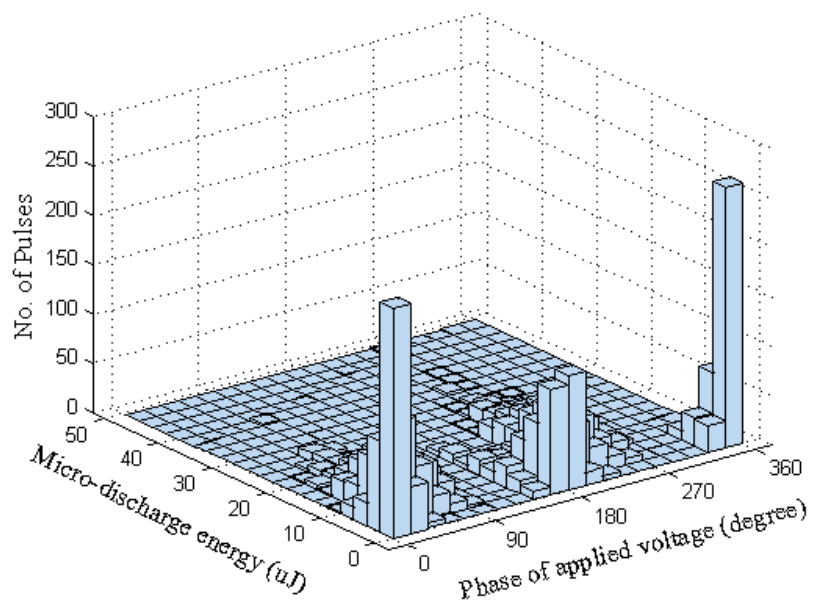

Fig. 8. Histogram of micro-discharge energy $W_{0}$ (at $0.5 \mathrm{~mm}$ gap length and 1 bar absolute)

is close to its peak $\left(90^{\circ}\right.$ and $\left.270^{\circ}\right)$, the micro-discharge energy becomes higher and the distribution is spread wider. The mean values of the parameters presented in Fig. 5, Fig. 6, Fig.7 and Fig. 8 are specified in TABLE 1.

TABLE 1

Mean values of electrical parameters of micro-discharges $(0.5 \mathrm{~mm}, 1 \mathrm{bar}$ absolute)

\begin{tabular}{ccc}
\hline Parameters & Positive & Negative \\
\hline$I_{p}$ & $1.0 \mathrm{~A}$ & $1.6 \mathrm{~A}$ \\
$t_{d}$ & $47 \mathrm{~ns}$ & $46 \mathrm{~ns}$ \\
$Q$ & $21 \mathrm{nC}$ & $32 \mathrm{nC}$ \\
$W_{0}$ & $7.2 \mu \mathrm{J}$ & $7.6 \mu \mathrm{J}$ \\
$n$ & 68 & 65 \\
\hline
\end{tabular}

\section{B. Comparison of the characteristics of negative filamentary current pulses for different gap lengths}

The negative filamentary current was selected for comparison at different gap lengths and gas pressures. Fig. 9 to Fig. 12 show the mean values and standard deviations of filamentary current pulse amplitude, duration, charge transfer and individual micro-discharge energy, respectively, for different 


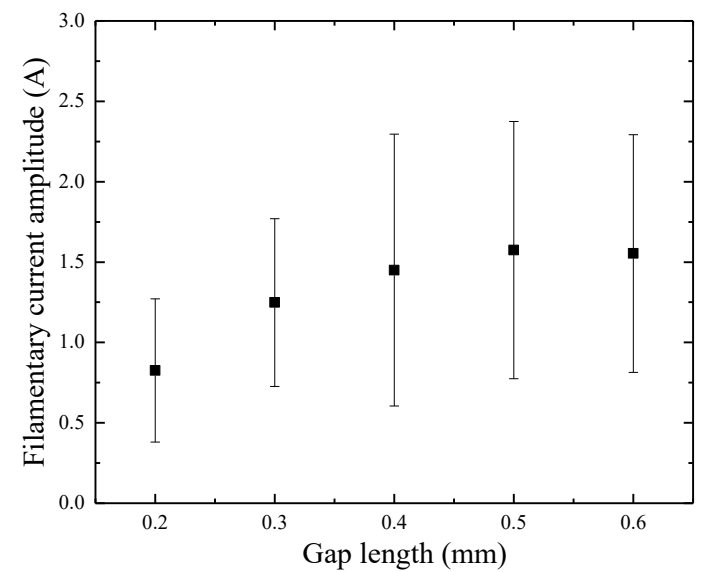

Fig. 9. Mean filamentary current amplitude for different gap lengths (at 1 bar absolute)

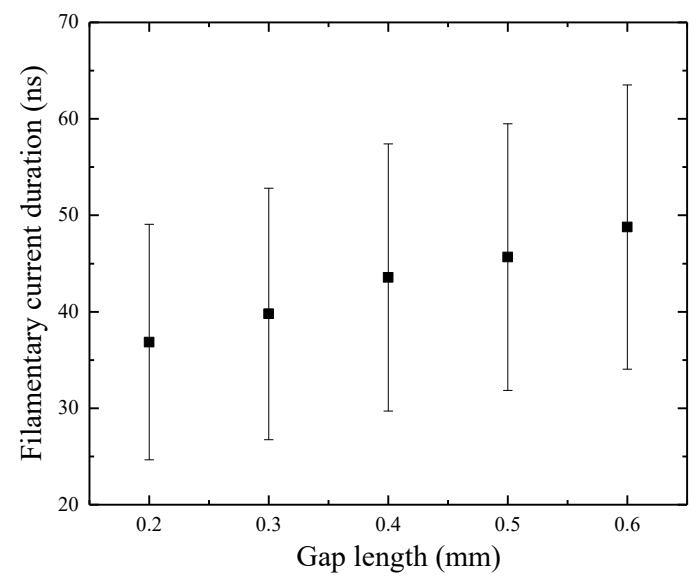

Fig. 10. Mean filamentary current duration for different gap lengths (at 1 bar absolute)

gap lengths at the same absolute pressure of 1 bar. The mean values generally increase with an increase in gap length. The mean filamentary current amplitude increases from $0.8 \mathrm{~A}$ with gap length of $0.2 \mathrm{~mm}$ to 1.6 A with gap length of $0.5 \mathrm{~mm}$, corresponding to an increase in mean charge transfer from 24 $\mathrm{nC}$ to $32 \mathrm{nC}$, as shown in Fig. 9 and Fig. 11 respectively. It was observed that the mean current amplitude increases with increasing gap length for shorter gaps $(0.2 \mathrm{~mm}$ to $0.4 \mathrm{~mm})$, before plateauing for longer gaps $(0.5 \mathrm{~mm}$ and $0.6 \mathrm{~mm})$. The mean current pulse duration increases from $37 \mathrm{~ns}$ with gap length of $0.2 \mathrm{~mm}$, to $49 \mathrm{~ns}$ at gap length of $0.6 \mathrm{~mm}$, and the micro-discharge energy increases from $5.6 \mu \mathrm{J}$ to $8.3 \mu \mathrm{J}$, as shown in Fig. 10 and Fig. 12 respectively.

\section{Comparison of the characteristics of micro-discharges at different gas pressures}

The mean values and standard deviations of filamentary current amplitude, duration, and transferred charge, and of individual micro-discharge energy, at different pressures with the same gap length of $0.5 \mathrm{~mm}$ are displayed in Fig. 13 to Fig. 16, respectively. It can be seen in Fig. 13 that the mean filamentary current amplitude increases from $1.6 \mathrm{~A}$ at 1 bar absolute to $2.6 \mathrm{~A}$ at 2 bar absolute, corresponding to an increase in charge transfer from $32 \mathrm{nC}$ to $54 \mathrm{nC}$, as shown in Fig. 15.

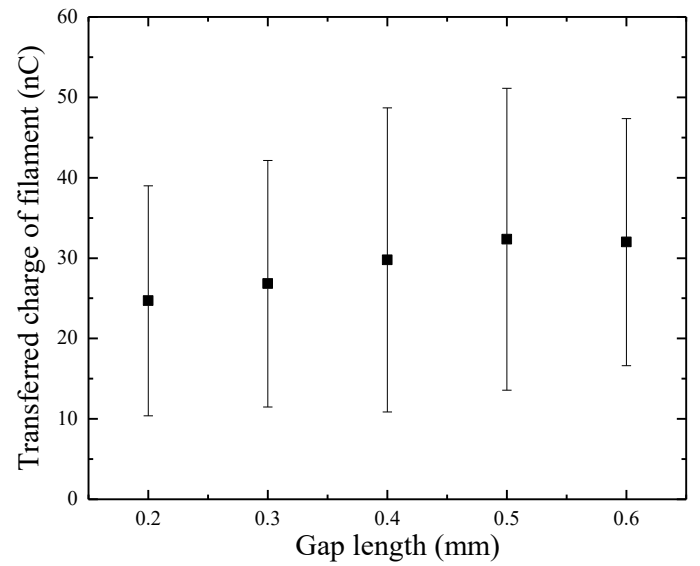

Fig. 11. Mean transferred charge of filament for different gap lengths (at 1 bar absolute)

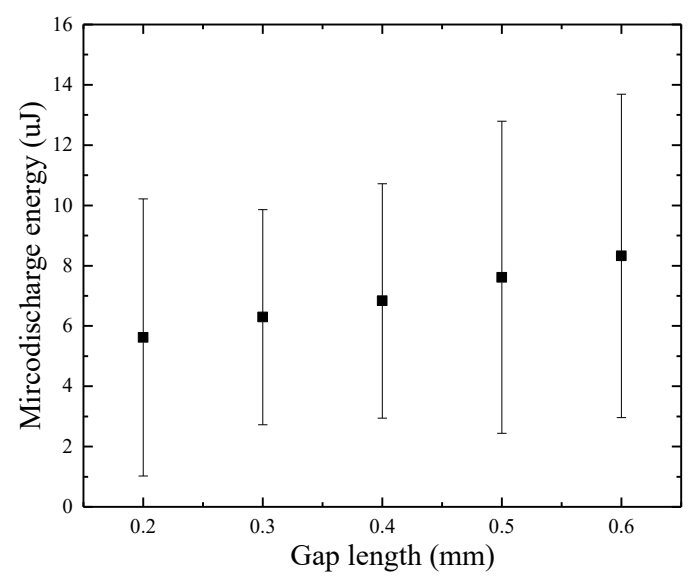

Fig. 12. Mean micro-discharge energy for different gap lengths (at 1 bar absolute)

Fig. 14 shows that the mean pulse duration is generally within the range from $45 \mathrm{~ns}$ to $50 \mathrm{~ns}$, demonstrating that the duration of the filamentary current pulse is not affected by gas pressure. Fig. 16 shows that the mean value of individual micro-discharge energy increases from $7.6 \mu \mathrm{J}$ at 1 bar absolute to $19.8 \mu \mathrm{J}$ at 2 bar absolute. Similar to the results for different gap lengths, the standard deviation of the distribution of these parameters was of the same order as the mean values, which means that the

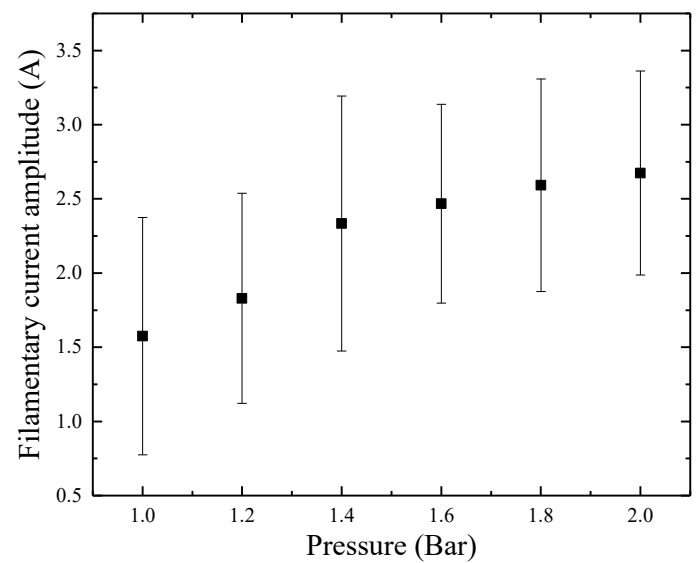

Fig. 13. Mean filamentary current amplitude at different (absolute) gas pressures (at $0.5 \mathrm{~mm}$ gap length) 


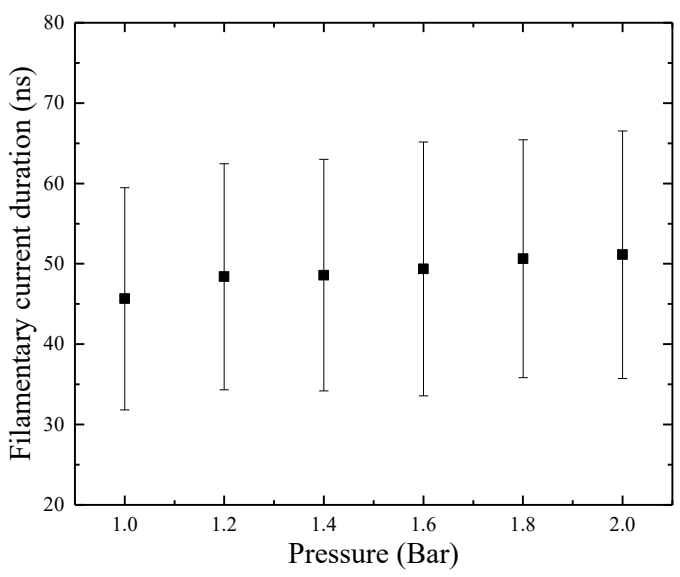

Fig. 14. Mean filamentary current duration at different (absolute) gas pressures (at $0.5 \mathrm{~mm}$ gap length)

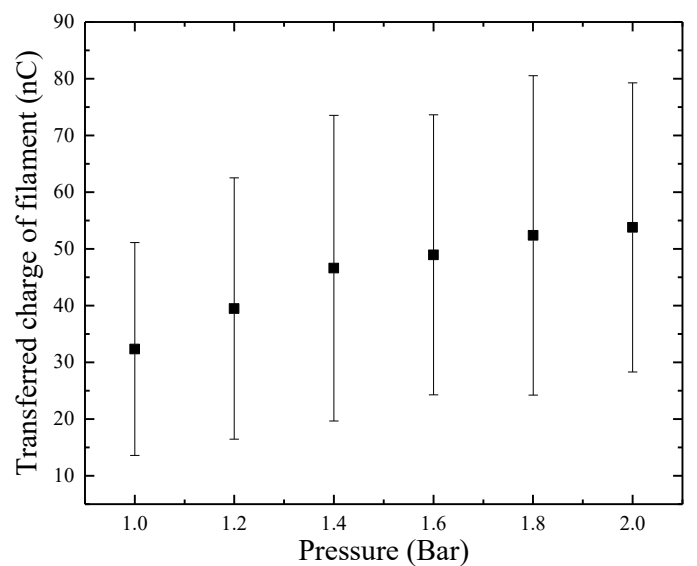

Fig. 15. Mean transferred charge of filament at different (absolute) gas pressures (at $0.5 \mathrm{~mm}$ gap length)

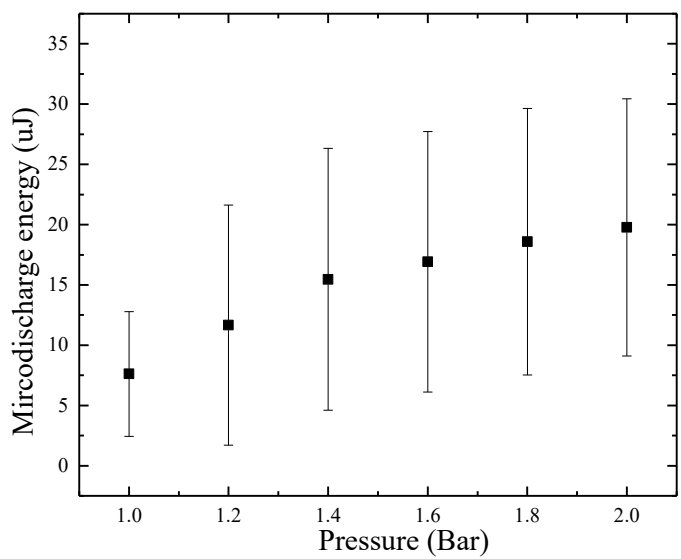

Fig. 16. Mean micro-discharge energy at different (absolute) gas pressures (at $0.5 \mathrm{~mm}$ gap length)

distribution of the filamentary current pulses was spread over the voltage phases.

\section{Ozone generation performance}

Fig. 17 shows that the ozone generation efficiency increased with increasing gap length from $0.2 \mathrm{~mm}$ to $0.5 \mathrm{~mm}$ and then saturated, with the highest ozone efficiency obtained being 166 $\mathrm{g} / \mathrm{kWh}$. It has been reported by a number of researchers that shorter gap length can result in better heat removal, which leads

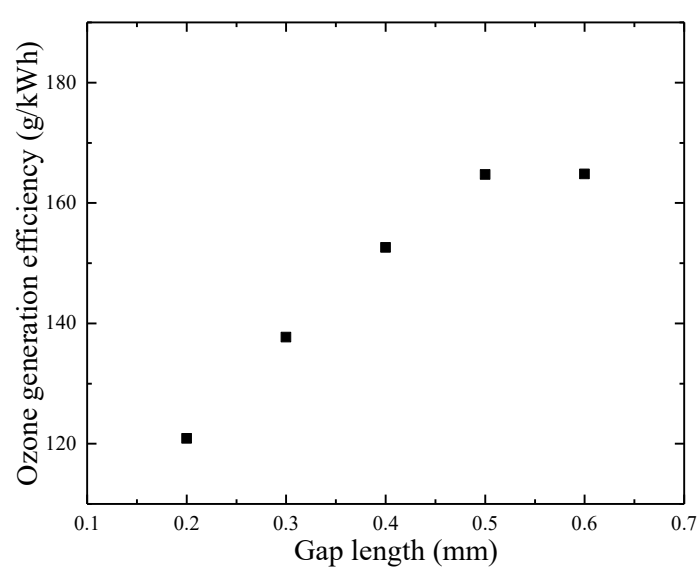

Fig. 17. Ozone generation efficiency with different gap lengths at 1 bar absolute, under applied voltage of $5.5 \mathrm{kV}$ at $5 \mathrm{kHz}$

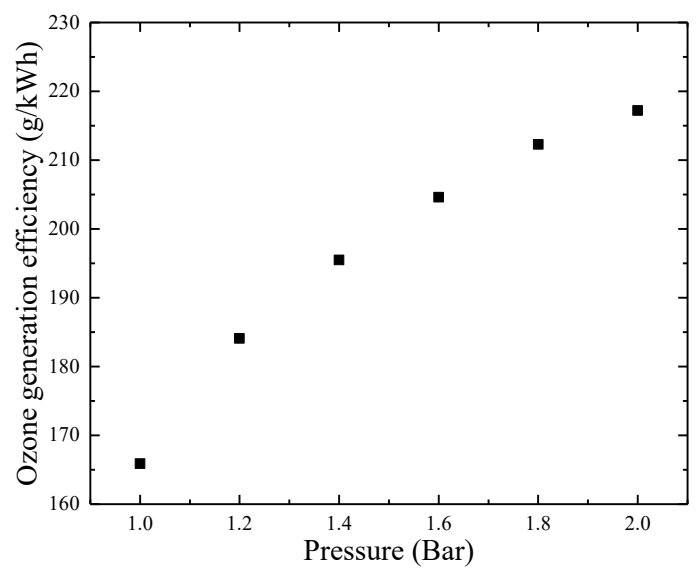

Fig. 18. Ozone generation efficiency with different (absolute) pressures for 0.5 $\mathrm{mm}$ gap length, under applied voltage of $5.5 \mathrm{kV}$ at $5 \mathrm{kHz}$

to increased ozone concentration and generation efficiency [13, 16]. In the present work, however, due to the application of 200 voltage cycles with the frequency of $5 \mathrm{kHz}$, the applied power duration is only $40 \mathrm{~ms}$, therefore the heating effect in the reactor can be neglected. Fig. 18 shows the dependence of ozone generation efficiency on gas pressure, demonstrating that the ozone efficiency increases with increasing gas pressure. The highest ozone generation efficiency of $217 \mathrm{~g} / \mathrm{kWh}$ was achieved at pressure of 2 bar absolute.

\section{DISCUSSION}

\section{A. Micro-discharges characteristics}

\section{1) Establishment of stationary dielectric barrier discharges}

Fig. 19 shows the waveform of the discharge current measured in the external circuit at the initial stage of the dielectric barrier discharges energized by 200 voltage cycles. From the discharge current waveform, it can be seen that the first cycle of the discharge current had a larger spike which is attributed to the transition of static gas state to gas discharge state. The accumulated charge on the barrier from the first cycle leaded to the establishment of stationary dielectric barrier discharge. From Fig. 19, it is shown that the discharge current waveform after the first cycle had a regular spike pattern. Therefore it can be considered that a stationary dielectric barrier discharge was established from the second cycle. 
Because this research work is focused on the low concentration ozone generation process without considering the temperature effect, the ozone generation efficiency is considered to be consistent once a stationary oscillatory discharge current was established.

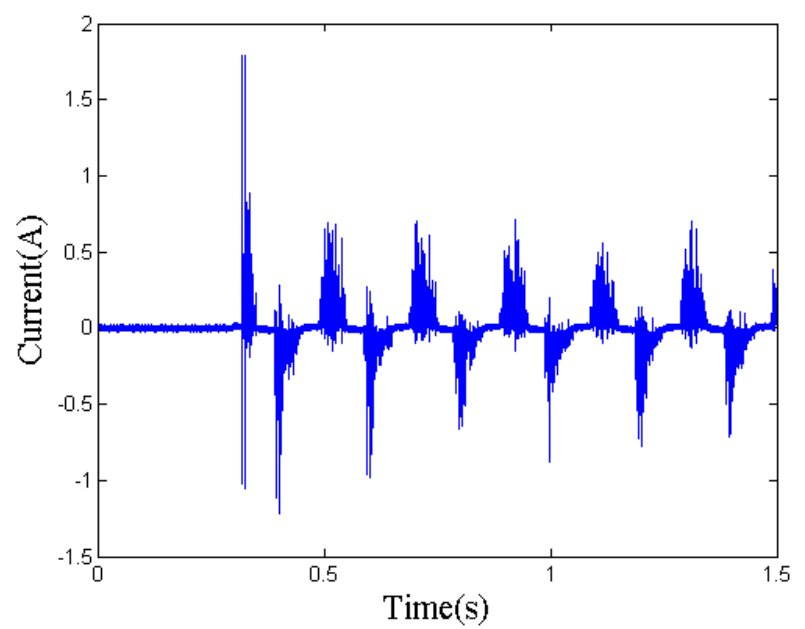

Fig. 19 Discharge current measured in the external circuit at the initial stage of the dielectric barrier discharges energized by 200 voltage cycles

2) Characteristics of filamentary current pulse distribution over one voltage cycle

From the histograms of filamentary current pulse amplitude (Fig. 5) and transferred charge (Fig. 7) of a single filament in Section III, a polarity effect of micro-discharges has been observed. The results show that the negative filamentary current is about 2 times higher than the positive filamentary current. This observation can be explained as follows. Before the positive discharge cycle starts, there are negative ions deposited on the surface of the dielectric barrier from the previous half-cycle. The accumulated negative charges can be considered as numerous individual point charges distributed over the dielectric barrier surface, which results in local electric field distortion. When the external electric field decreases to a certain value before zero volts, the local electric field around the accumulated point charge is high enough to initiate positive discharges, representative of a glow-like mode. When the positive discharge stops, the charges accumulated on the barrier surface are positive. The numerous positive point charges deposited on the surface result in a local electric-field distortion that is different from the negative point charge [15], which leads to a filamentary discharge mode. The filamentary discharge mode results in higher impulsive current than the glow-like discharge mode. This polarity effect was also found by T. Murata et al. in [17], who attributed it to the different mobility of electrons and ions accumulated on the glass surface.

It was found that, at the same voltage phase angle, the negative discharge current pulse amplitude values were dispersed. The average amplitudes around the voltage phase angles of $0^{\circ}$ or $180^{\circ}$, the initial stage of the discharge period, were relatively high compared to those around the voltage phase angles of $90^{\circ}$ or $270^{\circ}$, the final stage of the discharge period. This can be explained as follows. At the initial stage of the discharge period for each half-cycle, the charge accumulated on the dielectric surface from the previous half-cycle contributes to the development of a filament, and also the charge with opposite polarity transported in the filament at this stage has more space to develop, leading to a higher-amplitude current pulse. At the final stage of the discharge period, as the dielectric surface has accumulated a certain amount of charge, the charge with the same polarity transported in the filament has a limited space to develop due to the repulsion by nearby charge, which results in a lower current pulse amplitude.

It was also observed that the filamentary current in the gap is about 4 times higher than the induced pulsed current measured in the external circuit, which is mainly due to the high $d V_{a} / d t$ at the moment of discharge (see (1)). The calculated charge transferred per filament was about 10 times higher compared with that from a previous study [6] in which the rapidly changing voltage at the moment of discharge was not considered.

\section{3) The effects of discharge gap length and gas pressure on the micro-discharges}

Without considering the ionic current component in the filament current due to its small contribution [3], the current density, $j$, and the transferred charge, $Q_{c}$, of the filament can be calculated from (4) and (5), respectively:

$$
\begin{gathered}
j=e \cdot N_{e} \cdot v_{e} \\
Q_{c}=e \cdot F_{c} \cdot \int_{0}^{t_{d}} v_{e} \cdot N_{e} d t
\end{gathered}
$$

where $N_{e}, v_{\mathrm{e}}$ and $F_{\mathrm{c}}$ are the electron density, electron drift velocity, and the cross-section of the micro-discharge channel, respectively. Referring to (4) and (5), it can be deduced that the increase of filamentary current and transferred charge with increasing gap length and gas pressure results from the joint effects of the electron density and electron drift velocity at different gap lengths and gas pressures. The electron density and the electron drift velocity distribution are related to the reduced electric field $E / N[12,18]$. The $E / N$ at different gap lengths were calculated and plotted in Fig. 20. Fig. 20 shows that the $E / N$ decreases from $185 \mathrm{Td}$ to $130 \mathrm{Td}$ when the gap length increases from $0.2 \mathrm{~mm}$ to $0.6 \mathrm{~mm}$. It is interesting to find

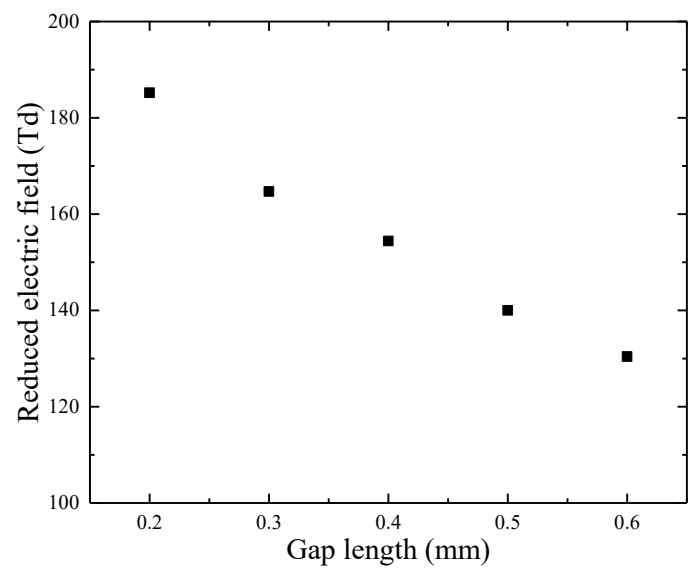

Fig. 20. The dependence of $E / N$ on gap length (at 1 bar absolute) 


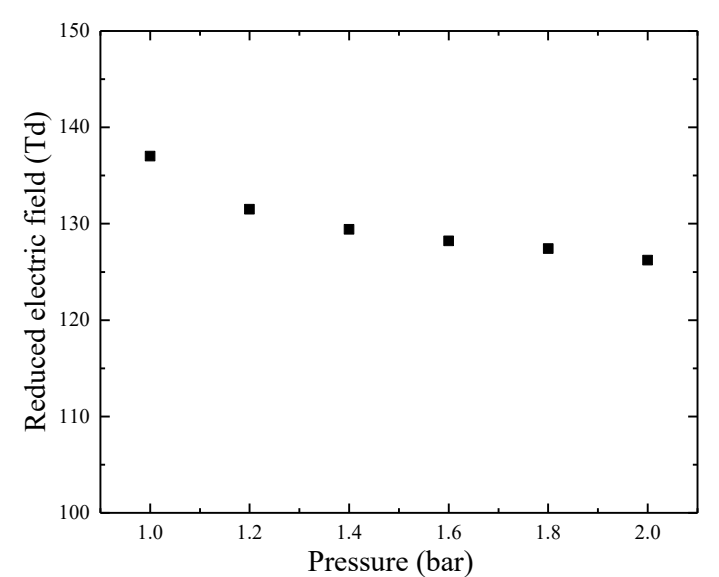

Fig. 21. The dependence of $E / N$ on (absolute) gas pressure (for $0.5 \mathrm{~mm}$ gap length)

that at lower electric field of $130 \mathrm{Td}$, the amplitude, transferred charge, and dissipated energy of filaments are higher compared with those at the higher electric field of $185 \mathrm{Td}$. The $E / N$ at different gas pressures was calculated and plotted in Fig. 21, which shows that the $E / N$ decreases slightly from $136 \mathrm{Td}$ at 1 bar absolute to $126 \mathrm{Td}$ at 2 bar absolute. Similar to the results obtained for different gap lengths, Fig. 21 demonstrates that the filamentary current amplitude, transferred charge, and individual micro-discharge energy are higher at lower levels of reduced electric field. The effect of $E / N$ on the distributions of electron density and electron drift velocity is complicated and requires further advanced plasma diagnostic techniques or application of the Particle-in-Cell method for numerical simulation $[19,20]$. The filamentary current pulse duration represents the time it takes to build up a counter electric field by accumulated charge that is high enough to terminate the discharge. From experimental results, it is known that when the discharge gap is longer, more time is needed to build up the counter electric field to make discharge extinguished but the filamentary current pulse duration time is not affected by increasing gas pressure.

The effects of discharge gap distance and gas pressure on the characteristics of micro-discharge have also been investigated by other researchers. Using a numerical method, Braun et al. reported that the radius of the micro-discharge increased with increasing gap length, and decreased with increasing gas pressure [21]. For longer gap length, with larger micro-discharge radius, the transferred charge of a single filament and the micro-discharge energy both increase. However, the pressure effect cannot be explained by this argument.

\section{B. Ozone performance}

Ozone generation results from two main opposite reactions: a reaction of ozone formation from oxygen and a reaction of ozone destruction. The key reactions involved in the ozone generation are shown in (6)-(12) [22]:

\section{1) Electronic dissociation of $\mathrm{O}_{2}$ :}

$$
e+O_{2} \stackrel{k_{1 a}}{\longrightarrow} O_{2}\left(\mathrm{~A}^{3} \sum_{u}^{+}\right) \rightarrow O\left({ }^{3} \mathrm{P}\right)+O\left({ }^{3} \mathrm{P}\right)+\mathrm{e}
$$

$$
e+O_{2} \stackrel{k_{1 b}}{\longrightarrow} O_{2}\left(\mathrm{~B}^{3} \Sigma_{u}^{-}\right) \rightarrow O\left({ }^{3} \mathrm{P}\right)+O\left({ }^{1} \mathrm{D}\right)+\mathrm{e}
$$

2) Formation of vibrational excited $\mathrm{O}_{3}$ :

$$
\begin{gathered}
O+O_{2}+M \stackrel{k_{2}}{\longrightarrow} O_{3}^{*}+M \\
\quad\left(M=[O],\left[O_{2}\right],\left[O_{3}\right]\right)
\end{gathered}
$$

3) $\mathrm{O}_{3}$ formation:

$$
\begin{aligned}
& O_{3}^{*}+O \stackrel{k_{3}}{\longrightarrow} O_{3}+O \\
& O_{3}^{*}+O_{2} \stackrel{k_{4}}{\longrightarrow} O_{3}+O_{2}
\end{aligned}
$$

4) $\mathrm{O}_{3}$ decomposition:

$$
\begin{gathered}
O+O_{3}^{*} \stackrel{k_{5}}{\longrightarrow} 2 O_{2} \\
e+O_{3} \stackrel{k_{6}}{\longrightarrow} O_{2}+O+\mathrm{e}
\end{gathered}
$$

In the above reactions, $k_{x}(x=1 \mathrm{a}, 1 \mathrm{~b}, 2 \ldots 6)$ is the reaction rate coefficient. In the oxygen discharges with the electric field strength $E$, assuming the portion of energy carried by electron from the input energy is $0.5[13,16]$, the ozone generation efficiency can be calculated by (13), based on the reactions from (4) to (10).

$$
\eta=\frac{\left(k_{1 a}+k_{1 b}\right)}{v_{d}(E / N)} \cdot \frac{1}{1+\frac{2 k_{5} x_{10}}{k_{3} x_{10}+k_{4}}} \quad O_{3} \text { molecules/ev (13) }
$$

Where $x_{10}$ is the oxygen atom concentration $\left(x_{10}=N[O] / N\left[O_{2}\right]\right)$ [3], $N$ is the initial concentration of $\mathrm{O}_{2}$ molecules (particles $\mathrm{cm}^{-3}$ ) and $v_{d}$ is the drift velocity of electrons. Referring to (13), it can be seen that the ozone generation efficiency is related to $E / N$. In the present work, different values of $E / N$ were achieved by adjusting the gap length and gas pressures. Based on the ozone generation efficiency measured at different conditions shown in Fig. 17 and Fig. 18, the ozone generation efficiency as a function of $E / N$ is presented in Fig. 22. It can be seen that the ozone generation efficiency decreases with increasing reduced electric field in the range from $126 \mathrm{Td}$ to $185 \mathrm{Td}$. This result is in agreement with the findings of Kitayama et al, who reported that the maximum ozone yield decreases gradually with increasing value of the reduced electric field strength, when $E / N>100 \mathrm{Td}$ [13]. It should be noted that the parameters of $k_{1 \mathrm{a}}$, $k_{1 \mathrm{a},} \lambda$ and $v_{\mathrm{d}}$ are all affected by $E / N$, and therefore changes in ozone generation efficiency at different $E / N$ are as a result of the joint effect of all of the parameters in (9).

From the statistical analysis of micro-discharge properties, it has been found that the micro-discharge energy is higher for longer gaps and larger gas pressures. Therefore, the ozone efficiency increases when the micro-discharge is more intense. The highest ozone generation efficiency of $217 \mathrm{~g} / \mathrm{kWh}$ occurred when the mean value of individual micro-discharge energy was $20 \mu \mathrm{J}$, at $E / N$ of $126 \mathrm{Td}$. Increased micro-discharge energy means that more oxygen atoms are produced in the 
micro-discharge channel, such that more ozone can be generated subsequently, based on (6), (7) and (8). Therefore, the ozone generation efficiency can be improved when the individual micro-discharge energy is increased. However there exists an optimum value of oxygen atom concentration that is beneficial for ozone generation, which is $10^{-4}$ when $E / N$ is about $100 \mathrm{Td}$, according to Elisasson et al.[3]. Whereas the oxygen atom concentration could not be measured in the present work, the optimum $E / N$ for ozone generation was found to be $126 \mathrm{Td}$. The highest ozone generation efficiency achieved by Elisasson et al. was $250 \mathrm{~g} / \mathrm{kWh}$ based on AC power supply [3]. Compared with that efficiency, the maximum ozone production efficiency we measured was about $13 \%$ lower.

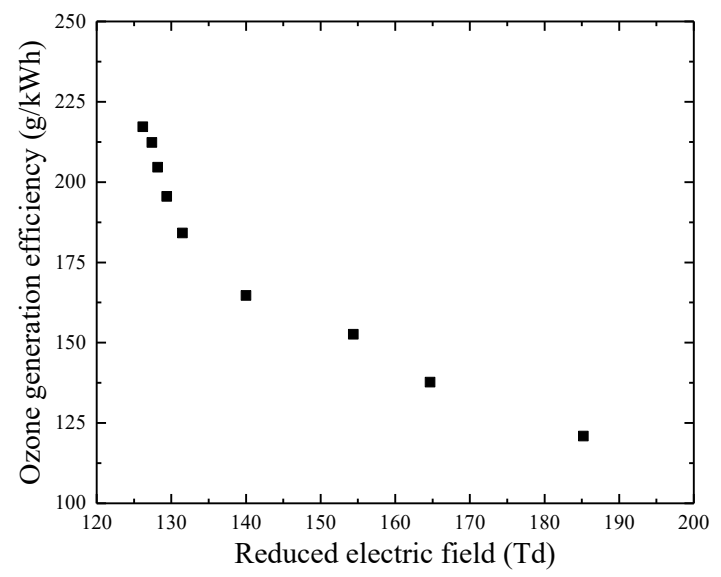

Fig. 22. Ozone generation efficiency at different $E / N$

\section{CONCLUSION}

In conclusion, the statistical characteristics of micro-discharge and ozone production under different gap lengths and gas pressures were studied in this work. A polarity effect was found, with negative discharge current twice higher than positive discharge current. The $d V_{a} / d t$ at the moment of discharge was considered and the filamentary current in the discharge gap was calculated. It was shown that the amplitude of the filamentary current was about 4 times higher than that of the external pulsed current.

Statistical analysis of representative pulses showed that the mean values of the amplitude, transferred charge and micro-discharge energy all increased as the gap length or gas pressure was increased. The filamentary current pulse duration increased with increasing gap length, but was not affected by gas pressure. The $E / N$ was calculated for different gap lengths and gas pressures, and it was observed that the filamentary current amplitude, transferred charge of an individual filament, and individual micro-discharge energy were higher at lower $E / N$, and also that the ozone generation efficiency was found to increase with decreasing $E / N$ in the range from $126 \mathrm{Td}$ to 185 $\mathrm{Td}$. In addition, the ozone generation efficiency was higher when the micro-discharge energy was higher. The maximum ozone production efficiency achieved in the work was 217 $\mathrm{g} / \mathrm{kWh}$ with a gap length of $0.5 \mathrm{~mm}, 2.0$ bar absolute pressure, and an applied voltage of $5.5 \mathrm{kV}$ at $5 \mathrm{kHz}$.

\section{REFERENCES}

[1] S. Jodzis and J. Petryk, "Gas Temperature in an Ozonizer. The Computer Modeling of an Actual Discharge System," IEEE Trans. Plasma Sci., vol. 39, no. 11, pp. 2020-2021, 2011. Available: http://ieeexplore.ieee.org/xpl/articleDetails.jsp?arnumber $=5957318 \&$ ne wsearch $=$ true\&queryText $=$ Gas $\% 20$ Temperature $\% 20 \mathrm{in} \% 20 \mathrm{an} \% 20$ Ozoni zer. $\% 20$ The $\% 20$ Computer $\% 20$ Modeling $\% 20$ of $\% 20$ an $\% 20$ Actual $\% 20$ Di scharge $\% 20$ System

[2] U. Kogelschatz, B. Eliasson, and W. Egli, "Dielectric-barrier discharges. Principle and applications," Journal de Physique IV (Colloque), vol. 7, pp. 47-66, $1997 . \quad$ Available: https://hal.archives-ouvertes.fr/jpa-00255561/document

[3] B. Eliasson, M. Hirth, and U. Kogelschatz, "Ozone synthesis from oxygen in dielectric barrier discharges," J. Phys. D: Appl. Phys., vol. 20, no. 11, pp. 1421-1437, $1987 . \quad$ Available: http://iopscience.iop.org/article/10.1088/0022-3727/20/11/010/pdf

[4] Z. Falkenstein and J. J. Coogan, "Microdischarge behaviour in the silent discharge of nitrogen - oxygen and water - air mixtures," J. Phys. D: Appl. Phys. , vol. 30, no. 5, pp. 817-825, 1997. Available: http://m.iopscience.iop.org/article/10.1088/0022-3727/30/5/015/pdf

[5] M. Heise, T. Lierfeld, O. Franken, and W. Neff, "Single filament charge transfer and UV-emission properties of a cascaded dielectric barrier discharge (CDBD) set-up," Plasma Sources Science and Technology, vol. 13, no. 12, pp. 351-358, 2004. Available: http://iopscience.iop.org/article/10.1088/0963-0252/13/2/023/meta

[6] J. Drimal, K. V. Kozlov, V. I. Gibalov, and V. G. Samoylovich, "On value of transferred charge in silent discharge under atmospheric pressure," Czech. J. Phys. B 38, pp. 159-165, 1988. Available: http://link.springer.com/article/10.1007\%2FBF01596492

[7] L. Shuhai and M. Neiger, "Electrical modelling of homogeneous dielectric barrier discharges under an arbitrary excitation voltage," $J$. Phys. D: Appl. Phys., vol. 36, pp. 3144-3150, 2003. Available: http://iopscience.iop.org/article/10.1088/0022-3727/36/24/009

[8] P. Reichen, A. Sonnenfeld, and P. Rudolf von Rohr, "Influence of increased velocity on the statistical discharge characteristics of He and air barrier discharges," J. Phys. D: Appl. Phys., vol. 43, p. 025207, 2010. Available: http://iopscience.iop.org/article/10.1088/0022-3727/43/2/025207/meta

[9] N. Jidenko, M. Petit, and J. P. Borra, "Electrical characterization of microdischarges produced by dielectric barrier discharge in dry air at atmospheric pressure," J. Phys. D: Appl. Phys., vol. 39, pp. 281-293, 2006.

Available: http://iopscience.iop.org/article/10.1088/0022-3727/39/2/008/meta

[10] U.Kogelschatz, "Advanced ozone generation," Process Technologies for Water Treatment, pp. 87-120, 1988. Available: http://link.springer.com/chapter/10.1007/978-1-4684-8556-1_9

[11] T. C. Manley, "The electric characteristics of the ozonator discharge," Trans. Electrochem. Soc.84, pp. 83-96, 1943. Available: http://jes.ecsdl.org/content/84/1/83.short

[12] A. Fridman and L. A. Kennedy, "Elementary processes of charged species in plasma," in Plasma Physics and Engineering, $2^{\text {nd }}$ ed, United States: Taylor and Francis Group, 2011, pp. 15-69.

[13] J. Kitayama and M. Kuzumoto, "Theoretical and experimental study on ozone generation characteristics of an oxygen-fed ozone generator in silent discharge," J. Phy. D: Appl. Phys., vol. 30, p. 2453, 1997. Available: http://iopscience.iop.org/article/10.1088/0022-3727/30/17/011/meta

[14] B. Eliasson and U. Kogelschatz, "Modeling and applications of silent discharge plasmas," IEEE Trans. Plasma Sci., vol. 19, pp. 309-323, 1991. Available:

http://ieeexplore.ieee.org/xpl/login.jsp?tp=\&arnumber=106829\&url=htt p\%3A\%2F\%2Fieeexplore.ieee.org\%2Fxpls\%2Fabs all.jsp\%3Farnumbe r\%3D106829

[15] E. Kuffel, W. S. Zaengl, and J. Kuffel, "Electrical breakdown in gases" in High Voltage Engineering: Fundamentals:, $2^{\text {nd }}$ ed., Newnes, 2000, pp. 281-366.

[16] S. Yagi and M. Tanaka, "Mechanism of ozone generation in air-fed ozonisers," J. Phys. D: Appl. Phys., vol. 20, pp. 1421-37, 1979. Available: http://iopscience.iop.org/article/10.1088/0022-3727/12/9/013/meta

[17] M. T. Takaaki Murata, Yuji Okita and Kouichi Yasuoka, "Polarity Effect of Silent Discharge," Ozone Science \& Engineering, vol. 17, pp. 575-586, 1995.

[18] I. Radu, R. Bartnikas, and M. R. Wertheimer, "Diagnostics and modelling of noble gas atmospheric pressure dielectric barrier discharges in homogeneous or diverging electric fields," J. Phys. D: Appl. Phys, vol. 
38, $\quad$ pp. $539-546, \quad 2005 . \quad$ Available: http://iopscience.iop.org/article/10.1088/0022-3727/38/4/005/meta

[19] H. C. Kim, F. Iza, S. S. Yang, M. Radmilović-Radjenović, and J. K. Lee, "Particle and fluid simulations of low-temperature plasma discharges: benchmarks and kinetic effects," J. Phys. D: Appl. Phys., vol. 38, pp. R283-R301, 2005. http://iopscience.iop.org/article/10.1088/0022-3727/38/19/R01/meta

[20] B. Hyowon, S. I. C, H. S. W, L. H.-J, and L. H. J, "Effect of gap distance on the characteristic of atmospheric pressure micro dielectric barrier discharges," Journal of the Korean Physical Society, vol. 59, p. 3453, 2011. Available: http://adsabs.harvard.edu/abs/2011JKPS...59.3453H

[21] D. Braun, U. kuchler, and G. Pietsch, "Microdischarges in air-fed ozonizers," J. Phys. D: Appl. Phys., vol. 24, p. 564, 1991. Available: http://iopscience.iop.org/article/10.1088/0022-3727/24/4/007/meta

[22] B. Eliasson and U. Kogelschatz, "Electron impact dissociation in oxygen," J. Phys. B: Atomic and Molecular Physics, vol. 19, p. 1241, 1986.

http://iopscience.iop.org/article/10.1088/0022-3700/19/8/018/meta

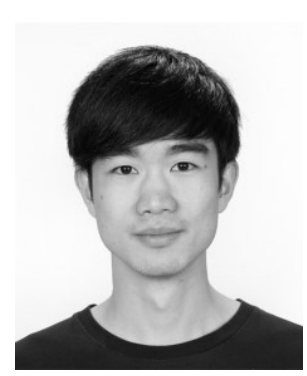

Guangming Huang received the B.Eng. $\left(1^{\text {st }}\right.$ Class $)$ degree in electronic and electrical engineering from the University of Strathclyde, Glasgow, UK. From 2010, he has been working toward the $\mathrm{Ph} . \mathrm{D}$. degree in the high voltage technology group in the department of electronic and electrical engineering at the University of Strathclyde. His Ph.D. research is focused on non-thermal plasma discharges for the ozone generation.

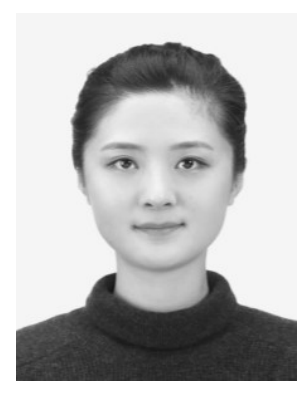

Yingjia Zhou was born in Jiangsu Province, China, 1990. She received the Msc (Merit) Power System Engineering from University College London in 2013. Before that, she received B. Eng (First Class Hons) Electronic and Electrical Engineering from University of Strathclyde in 2012. From 2013, she has been a Ph.D. student under High Voltage Technologies group in the department of electronic and electrical engineering at University of Strathclyde. Her current research interest includes non-thermal plasma discharge applications such as ozone generation. Recently, she is working on the process of Dielectric Barrier Discharge.

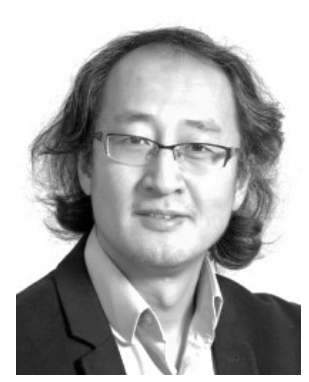

Tao Wang received the B.Eng and M.Sc degrees from Northeast China Dianli University (China) in 1993 and 1996 respectively, and the Ph.D. degree from the University of Strathclyde (Glasgow, UK) in 2005. He then joined the Newland Group as a research fellow developing industrial ozone generator. He joined the Department of Electronic and Electrical Engineering of University of Strathclyde as a lecturer in 2010. His research interests include non-thermal plasma and their applications in gas synthesis, plasma water interactions, water purification and advanced oxidation process.

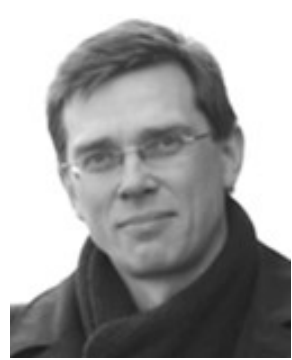

Igor V. Timoshkin (M'07-SM'14) received a degree in physics from the Moscow State University (Russia) in 1992, and the Diploma and the Ph.D. degree from the Imperial College of Science, Technology and Medicine, (London, UK) in 2001. After graduation from MSU he worked as a Researcher at Moscow State Agro-Engineering University, and then at the Institute for High Temperatures of Russian Academy of Sciences before moving to ICSTM in 1997. He joined the Department of Electronic and Electrical Engineering of the University of Strathclyde (Glasgow, UK) in 2001 where he became a Senior Lecturer in 2011. His research interests include properties of solid and liquid dielectric materials, electronics of plasma discharges in condensed media, practical applications of electro-hydraulic and high-power ultrasound pulses, bio-dielectrics and effects of electromagnetic fields on biological objects.

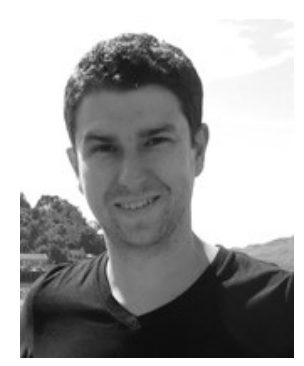

Mark P. Wilson (M'10) was born in Stranraer, Scotland, in 1982. He received the B.Eng. (with honours), M.Phil., and Ph.D. degrees in electronic and electrical engineering from the University of Strathclyde, Glasgow, U.K., in 2004, 2007, and 2011, respectively. He is presently working as a Teaching Associate at the University of Strathclyde, where he continues to investigate surface flashover of solids immersed in insulating oil. Mark is a member of the IEEE Nuclear and Plasma Sciences Society, from whom he received a Graduate Scholarship Award in 2011, the IEEE Dielectrics and Electrical Insulation Society, and the IET.

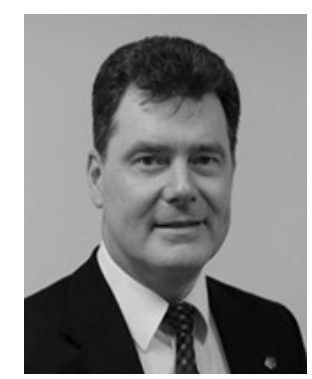

Scott J. MacGregor (M'95-SM'14) received the B.Sc. and $\mathrm{Ph}$.D. degrees from the University of Strathclyde, Glasgow, U.K., in 1982 and 1986, respectively. He became a Pulsed Power Research Fellow in 1986 and a Lecturer in pulsedpower technology in 1989. In 1994, he became a Senior Lecturer, with a promotion to Reader and Professor of High Voltage Engineering, in 1999 and 2001, respectively. From 2010 he became a Dean of the Engineering Faculty of the University of Strathclyde. His research interests include high-voltage pulse generation, high-frequency diagnostics, high-power repetitive switching, high-speed switching, electronic methods for food pasteurization and sterilization, generation of high-power ultrasound (HPU), plasma channel drilling, pulsed-plasma cleaning of pipes, and simulation of oil wells with HPU. 


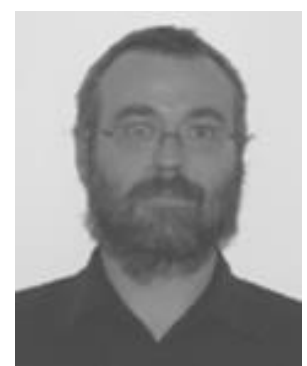

Martin J. Given (M'99-SM'11) is currently a Senior Lecturer in the Department of Electronic and Electrical Engineering at the University of Strathclyde. He received a degree in physics from the University of Sussex in 1981 and $\mathrm{a} \mathrm{PhD}$ in electronic and electrical engineering from the University of Strathclyde in 1996. His research interests include, ageing processes and condition monitoring in solid and liquid insulation systems, high speed switching and pulse power. 